\title{
Intensive Rehabilitation Therapy Following Brain Tumor Surgery: A Pilot Study of Effectiveness and Long-Term Satisfaction
}

\author{
Junghoon Yu, $\mathrm{MD}^{1}$, Youngsu Jung, $\mathrm{MD}^{1}$, Joonhyun Park, $\mathrm{MD}^{1}$, Jong Moon Kim, $\mathrm{MD}^{1,2}$, \\ Miri Suh, $\mathrm{MD}^{1,2}$, Kyung Gi Cho, $\mathrm{MD}^{3}$, MinYoung Kim, MD, $\mathrm{PhD}^{1,2}$
}

${ }^{1}$ Department of Rehabilitation Medicine, CHA Bundang Medical Center, CHA University School of Medicine, Seongnam;

${ }^{2}$ Rehabilitation and Regeneration Research Center, CHA University, Seongnam; ${ }^{3}$ Department of Neurosurgery, CHA Bundang Medical Center, CHA University School of Medicine, Seongnam, Korea

\begin{abstract}
Objective To evaluate the effectiveness of intensive rehabilitation to support recovery of neurological function after brain tumor surgery and assess long-term satisfaction.

Methods This retrospective study included patients with neurological impairment after brain tumor surgery who underwent intensive rehabilitation therapy between December 2013 and May 2017. To assess effectiveness of rehabilitation, functional outcomes (motor, cognition, and activities of daily living [ADL]) were compared between brain tumor group and a control group enrolling stroke patients who received equivalent rehabilitation during the study period. Long-term satisfaction with rehabilitation was evaluated by surveying family caregivers.

Results This study included 21 patients with benign brain tumor, 14 with malignant brain tumor, and 108 with stroke. Significant and similar improvement in motor, cognition, and ADL function were noted in both the brain tumor group and the stroke group. Malignancy status did not influence the extent of functional improvement. According to medical records and surveys, 9 (69.2\%) patients with malignant tumor and 2 (11.8\%) with benign tumor had expired by the time of the survey. Most family caregivers confirmed that rehabilitation was effective for functional improvement $(>60 \%)$, expressing overall satisfaction and stating they would recommend such therapy to patients with similar conditions (approximately 70\%).

Conclusion Intensive rehabilitation may help promote functional improvement following brain tumor surgery regardless of malignancy compared with stroke patients. Family caregivers expressed overall satisfaction with rehabilitation at long-term follow-up. These findings support the provision of intensive rehabilitation therapy for neurologic function recovery following brain tumor surgery.
\end{abstract}

Keywords Brain neoplasms, Treatment outcome, Satisfaction, Neurological rehabilitation

Received August 16, 2018; Accepted October 17, 2018

Corresponding author: MinYoung Kim

Department of Rehabilitation Medicine, CHA Bundang Medical Center, CHA University School of Medicine, 59 Yatap-ro, Bundang-gu, Seongnam 13496, Korea. Tel: +82-31-780-1872, Fax: +82-31-780-3449, E-mail: kmin@cha.ac.kr

ORCID: Junghoon Yu (http://orcid.org/0000-0001-6039-5364); Youngsu Jung (http://orcid.org/0000-0001-8173-736X); Joonhyun Park (http://orcid. org/0000-0002-6782-670X); Jong Moon Kim (http://orcid.org/0000-0002-8684-8736); Miri Suh (http://orcid.org/0000-0003-1759-9198); Kyung Gi Cho (http://orcid.org/0000-0001-7855-2719); MinYoung Kim (http://orcid.org/0000-0001-5481-2985).

(c) This is an open-access article distributed under the terms of the Creative Commons Attribution Non-Commercial License (http://creativecommons.org/ licenses/by-nc/4.0) which permits unrestricted noncommercial use, distribution, and reproduction in any medium, provided the original work is properly cited. Copyright (c) 2019 by Korean Academy of Rehabilitation Medicine 


\section{INTRODUCTION}

Brain tumor is a major cause of disability. The prevalence of intracranial tumors is increasing [1]. Due to technological advancements in surgery, chemotherapy, and radiotherapy, the survival rate of patients with brain tumors has increased substantially [2-4], creating an increasing need to address the remaining neurological impairment and restore the quality of life of brain tumor survivors $[5,6]$. Rehabilitation therapy after brain tumor removing or reducing treatments has gained increasing attention [2,5]. However, neuro-surgeons, neuro-oncologists, and even physiatrists tend to overlook the potential of rehabilitation following brain tumor surgery considering the relatively low rate of survival and high rate of recurrence in this patient population [4,7-9].

In 1978, Lehmann et al. [10] established the need for rehabilitation in cancer patients. Since then, several studies have been conducted about the impact of rehabilitation on functional outcomes and recovery of neurological impairment in patients with brain tumors [5,11-17]. As stroke and brain tumors are associated with similar symptoms (e.g., cognitive impairment, motor dysfunction, sensory dysfunction, ataxia, dysphagia, aphasia, and visual perceptional deficits) $[18,19]$, it is unsurprising that the effectiveness of rehabilitation therapy in terms of various outcome measures is similar between these two patient populations $[2,11,16,20]$.

While the short-term effectiveness of rehabilitation therapy in brain tumor patients has been recognized, it remains controversial whether rehabilitation is meaningful and necessary if the survival rate is low and the recurrence rate is high. So far, no studies have addressed this important question. In the absence of an objective indicator of the potential need and benefit of rehabilitation for patients with brain tumors, long-term satisfaction of family caregivers may serve as a surrogate measure in the context of evolution of neurologic function throughout patients' remaining life.

In this study, we aimed to (1) comparatively assess the effectiveness of intensive rehabilitation therapy for improving neurological deficit following brain tumor surgery, taking functional outcomes of rehabilitation in stroke patients as reference as the usefulness of intensive rehabilitation in stroke patients has already been validated [11-20]; and (2) gauge the opinion of family caregivers regarding their long-term satisfaction with intensive rehabilitation taking patients' survival or functional status into account.

\section{MATERIALS AND METHODS}

This study was approved by the Institutional Review Board of CHA Bundang Medical Center (No. 2018-01002-001). We retrospectively reviewed medical records maintained at a single university hospital rehabilitation center and identified patients with neurological impairment after brain tumor resection surgery or stroke who were admitted for intensive rehabilitation between December 2013 and May 2017.

To minimize bias, all patients fulfilling the inclusion/ exclusion criteria during the study period were enrolled in this study. Inclusion criteria were (1) brain tumor or stroke defined as brain lesions on computed tomography or magnetic resonance imaging; (2) admission for rehabilitation within $<4$ months since brain tumor resection surgery or stroke onset; (3) age >18 years; and (4) discharged for $>1$ year. Exclusion criteria were (1) combined neurodegenerative disease; and (2) persistent minimally conscious state or vegetative status. Patients with brain tumor were divided into subgroups based on malignancy status (benign and malignant) defined according to the World Health Organization classification [21].

The rehabilitation center implements a routine process for baseline functional assessment soon after admission and follow-up assessment at $>1$ month after admission. Standard rehabilitation therapy consisted of 1 hour of physical therapy and 1 hour of occupational therapy, with sessions conducted 5 days/week during hospitalization. The aim of rehabilitation therapy in patients with neurological deficit was to facilitate their self-management ability. The rehabilitation protocol was developed by the clinical therapy team.

Physical therapy focused on range-of-motion exercise, progressive resistive exercise, balance training, endurance training, and gait training. Occupational therapy focused on cognitive ability enhancement, upper extremity proprioceptive neuromuscular facilitation, and activities of daily living (ADL) training. Outcome analysis was conducted based on the rehabilitation center's records documenting results of baseline assessment conducted within 5 days of admission and results of follow-up assessment. 
Functional assessments was conducted using the following tools: Fugl-Meyer Assessment (FMA) index, Berg Balance Scale (BBS) score, Korean version of the Modified Barthel Index (K-MBI) score, Korean Mini-Mental State Examination (K-MMSE) score, and intelligence quotient (IQ) score on the Korean version of the Wechsler Adults Intelligence Scale. Among brain tumor patients, the Eastern Cooperative Oncology Group (ECOG) score was also evaluated to confirm the functional level. In our rehabilitation center, reliability tests for these above-mentioned scores are performed regularly, with all intraclass correlation coefficients for inter-rater reliability maintained at $>0.9$. Demographic and clinical characteristics of patients were obtained from medical records. For patients with brain tumors, additional information was collected, including histologic type, location of the tumor, recurrence status (primary or recurred tumor), and duration of surgery.

Long-term satisfaction with rehabilitation therapy was assessed via telephone survey of family caregivers of patients with brain tumors. The survey was conducted after May 2018, when more than 1 year had passed since the discharge of the last patient enrolled in this study. The survey contained six items that evaluated the opinion of caregivers on whether rehabilitation brought improvement in (1) motor function, (2) cognition, (3) ADL; (4) whether the intensity of rehabilitation therapy was adequate; (5) their overall satisfaction with rehabilitation; and (6) whether they would recommend such rehabilitation to other patients with similar conditions (Supplementary Table S1). Other long-term data (survival status, date of death, and cause of death) were obtained from medical records and telephone surveys. Among survivors, change in functional status since discharge was assessed subjectively for motor, cognition, and ADL function (improved, similar, or worsened).

The usefulness of rehabilitation therapy has already been validated in stroke patients [11-20]. In this study, we hypothesized that rehabilitation would be equally effective in brain tumor patients. Thus, the sample size estimate was based on the hypothesis that effects of rehabilitation therapy would not differ between the brain tumor group and the stroke group. A power calculation was conducted using G*Power version 3.1.9.2 (http:// www.gpower.hhu.de/). Setting alpha to 0.05 and beta to 0.20 , the minimum sample size was calculated to be 64 subjects per group. However, as the number of patients admitted for intensive rehabilitation therapy following brain tumor surgery was low, the final size of the brain tumor group ( $\mathrm{n}=35)$ was smaller than the estimated sample size.

The Statistical Package for the Social Sciences (SPSS) version 21.0 for Windows (IBM, Armonk, NY, USA) was used for all data analyses. Independent t-test and chisquare test were used to compare brain tumor and stroke groups of patients in terms of demographic and baseline characteristics. The significance of functional improvements in each group was confirmed using the Wilcoxon signed-rank test which was applied for each outcome parameter. Mann-Whitney U test was used to compare outcomes of rehabilitation between brain tumor and stroke groups as well as between benign and malignant tumor subgroups. In addition to effectiveness of rehabilitation for each outcome parameter, the efficiency of rehabilitation was calculated as the amount of change divided by the length of rehabilitation. It represents the average degree of improvement that can be achieved daily through rehabilitation therapy. Multivariate linear regression analysis was used to identify baseline predictors of rehabilitation outcome in brain tumor patients. The survival rate of the brain tumor group was analyzed using the Kaplan-Meier survival curve. Chi-square test was performed to detect associations of overall satisfaction with other variables such as long-term improvements in motor, cognition, and ADL function; long-term survival status; and malignancy status on pathology examination. Statistical significance was set at $\mathrm{p}<0.05$.

\section{RESULTS}

\section{General patient characteristics}

Of 35 brain tumor patients included in this study, 21 had benign tumor and 14 had malignant tumor. As controls, we enrolled 108 patients with stroke (Fig. 1). The mean age was 57.6 years in the brain tumor group and 60.9 years in the stroke group. Baseline demographics including age, sex, lesion side, and the length of inpatient rehabilitation were similar between the two groups (Table 1). Baseline characteristics were also similar between benign and malignant tumor subgroups. Among brain tumor patients, 24 (68.6\%) had primary tumors while 11 (31.4\%) had recurrent tumor. 


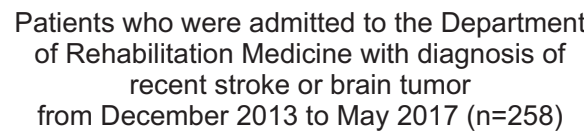

Patients who were admitted to the Departmen of Rehabilitation Medicine with diagnosis of recent stroke or brain tumor from December 2013 to May $2017(n=258)$

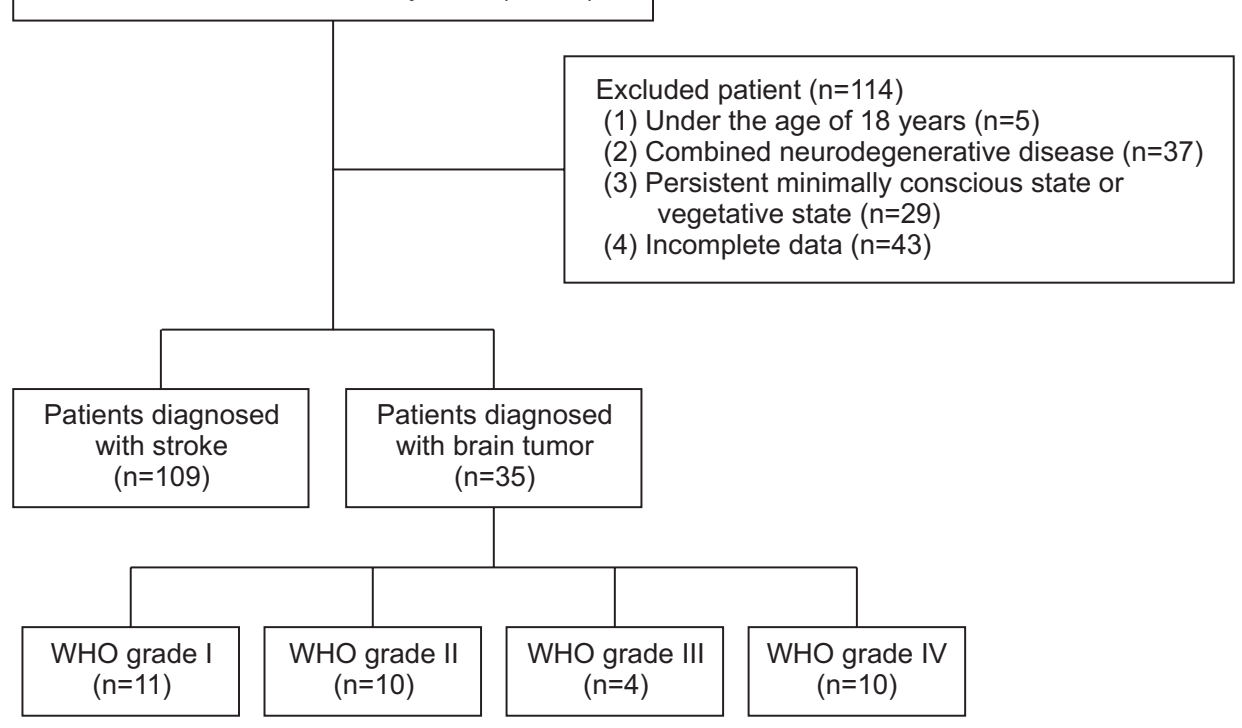

Fig. 1. Recruitment flowchart detailing the number of participants included and excluded. WHO, World Health Organization.

Table 1. Demographic and disease-related characteristics

\begin{tabular}{|c|c|c|c|}
\hline & Brain tumor group $(n=35)$ & Stroke group $(n=108)$ & p-value \\
\hline Age (yr) & $57.6 \pm 13.2$ & $60.9 \pm 11.6$ & 0.275 \\
\hline Gender & & & 0.890 \\
\hline Male & $18(51.4)$ & $57(52.8)$ & \\
\hline Female & $17(48.6)$ & $51(47.2)$ & \\
\hline Lesion location & & & 0.527 \\
\hline Right hemisphere & $16(45.7)$ & $47(43.5)$ & \\
\hline Left hemisphere & $14(40.0)$ & $41(38.0)$ & \\
\hline Cerebellum & $2(5.7)$ & $9(8.3)$ & \\
\hline Brain stem & $3(8.6)$ & $11(10.2)$ & \\
\hline \multicolumn{4}{|l|}{ Tumor etiology (WHO grade) } \\
\hline I (benign tumor) & $11(31.4)$ & NA & - \\
\hline II (benign tumor) & $10(28.6)$ & & \\
\hline III (malignant tumor) & $4(11.4)$ & & \\
\hline IV (malignant tumor) & $10(28.6)$ & & \\
\hline \multicolumn{4}{|l|}{ Recurrence } \\
\hline Primary & $24(31.4)$ & & \\
\hline Recurrent & $11(68.6)$ & & \\
\hline Length of rehabilitation (day) & $37.2 \pm 11.9$ & $36.4 \pm 6.7$ & 0.759 \\
\hline
\end{tabular}

Values are presented as number or mean \pm standard deviation or number of patients (\%).

Mean value and the percentage of each groups were compared by independent $t$-test.

Continuous data were tested by independent $\mathrm{t}$-test and categorical data were tested by chi-square test.

WHO, World Health Organization; NA, not applicable. 
Table 2. Brain tumor characteristics

\begin{tabular}{|c|c|c|c|}
\hline & $\begin{array}{c}\text { Benign brain tumor } \\
(n=21)\end{array}$ & $\begin{array}{l}\text { Malignant brain tumor } \\
(\mathrm{n}=14)\end{array}$ & p-value \\
\hline Lesion location & & & 0.537 \\
\hline Right frontal & $4(19.0)$ & $3(21.4)$ & \\
\hline Right temporal & $2(9.5)$ & $2(14.3)$ & \\
\hline Right parietal & $0(0)$ & $1(7.1)$ & \\
\hline Right hypothalamus & $1(4.8)$ & $0(0)$ & \\
\hline Right thalamus & $0(0.0)$ & $1(7.1)$ & \\
\hline Left frontal & $1(4.8)$ & $1(7.1)$ & \\
\hline Left temporal & $4(19.0)$ & $2(14.3)$ & \\
\hline Left parietal & $1(4.8)$ & $1(7.1)$ & \\
\hline Left thalamus & $0(0)$ & $1(7.1)$ & \\
\hline Left cingulate gyrus & $0(0)$ & $1(7.1)$ & \\
\hline Left lateral ventricle & $3(14.3)$ & $0(0.0)$ & \\
\hline Cerebellum & $1(4.8)$ & $1(7.1)$ & \\
\hline Brain stem & $3(14.3)$ & $0(0)$ & \\
\hline Skull base & $1(4.8)$ & $0(0)$ & \\
\hline Lesion size, long axis $(\mathrm{cm})$ & & & 0.537 \\
\hline $2-3$ & $3(14.3)$ & $2(14.3)$ & \\
\hline $3-4$ & $7(33.3)$ & $4(28.6)$ & \\
\hline $4-5$ & $6(28.6)$ & $2(14.3)$ & \\
\hline $5-6$ & $4(19.0)$ & $3(21.4)$ & \\
\hline $6-7$ & $1(4.8)$ & $3(21.4)$ & \\
\hline Treatment modality & & & $<0.001^{* * *}$ \\
\hline Surgery & $13(61.9)$ & $0(0)$ & \\
\hline Surgery+chemotherapy & $1(4.8)$ & $2(14.3)$ & \\
\hline Surgery+radiotherapy & $7(33.3)$ & $4(28.6)$ & \\
\hline Surgery+chemotherapy+radiotherapy & $0(0)$ & $8(57.1)$ & \\
\hline \multicolumn{4}{|l|}{ Recurrence } \\
\hline Primary & $16(76.2)$ & $8(57.1)$ & $<0.001^{* * *}$ \\
\hline Recurrent & $5(23.8)$ & $6(42.9)$ & \\
\hline \multicolumn{4}{|l|}{ Initial ECOG scale } \\
\hline 1 & $2(9.5)$ & $0(0)$ & 0.071 \\
\hline 2 & $4(19.0)$ & $3(14.3)$ & \\
\hline 3 & $7(33.3)$ & $9(42.9)$ & \\
\hline 4 & $8(38.1)$ & $2(9.5)$ & \\
\hline
\end{tabular}

Values are presented as number of patients (\%).

Benign brain tumor subgroup included atypical meningioma, atypical neurocytoma, chordoid glioma, chordoma, craniopharyngioma, hemangioblastoma, meningothelial meningioma, pilocytic astrocytoma, pleomorphic xanthoastrocytoma, reactive gliosis and schwannoma. Malignant brain tumor subgroup included anaplastic astrocytoma and glioblastoma.

ECOG, Eastern Cooperative Oncology Group.

*** $\mathrm{p}<0.001$, when continuous data were tested by independent $\mathrm{t}$-test, and categorical data were tested by chi-square test. 
There were no significant differences in ECOG score, tumor location, or lesion size between benign and malignant tumor subgroups (Table 2). However, these two subgroups differed in preference of treatment modality and rate of recurrence.

\section{Rehabilitation outcomes}

Significant improvement in FMA, BBS, K-MBI, KMMSE, and IQ scores were noted within both brain tumor and stroke groups (each p<0.001) (Table 3 ) and within benign and malignant brain tumor subgroups (each $\mathrm{p}<0.05$ ) (Table 4). There was no significant betweengroup or between-subgroup difference in terms of effectiveness (Tables 3, 4) or efficiency (Fig. 2A, 2B).

Among brain tumor patients, the improvement in ECOG score was not significantly affected by malignancy status (Table 4). However, the final rehabilitation outcome was significantly correlated with the initial functional status (Table 5). In other words, brain tumor patients with better baseline function for a specific parameter had higher improvement for this parameter. Specifically, improvement in FMA, BBS, and K-MBI scores

Table 3. Rehabilitation outcomes for brain tumor and stroke groups

\begin{tabular}{|c|c|c|c|c|c|c|c|}
\hline & \multicolumn{3}{|c|}{ Brain tumor group $(n=35)$} & \multicolumn{3}{|c|}{ Stroke group $(n=108)$} & \multirow{2}{*}{$\begin{array}{c}\text { p-value } \text { a) }^{\text {) }} \\
\text { (between } \\
\text { groups) }\end{array}$} \\
\hline & Baseline & Discharge & $\begin{array}{l}\text { p-value } \\
\text { (within } \\
\text { groups) }\end{array}$ & Baseline & Discharge & $\begin{array}{l}\text { p-value } \\
\text { (within } \\
\text { groups) }\end{array}$ & \\
\hline FMA score & $34.5 \pm 22.2$ & $49.1 \pm 17.5$ & $<0.001^{* * *}$ & $30.1 \pm 21.6$ & $41.8 \pm 21.0$ & $<0.001^{* * *}$ & 0.737 \\
\hline BBS score & $17.0 \pm 15.9$ & $35.6 \pm 17.7$ & $<0.001^{* * *}$ & $20.7 \pm 18.0$ & $35.2 \pm 17.7$ & $<0.001^{* * *}$ & 0.929 \\
\hline K-MBI score & $36.9 \pm 18.5$ & $58.3 \pm 20.1$ & $<0.001^{* * *}$ & $38.6 \pm 21.0$ & $56.7 \pm 23.2$ & $<0.001^{* * *}$ & 0.447 \\
\hline K-MMSE score & $17.3 \pm 10.1$ & $23.1 \pm 7.6$ & $<0.001^{* * *}$ & $16.7 \pm 8.2$ & $21.1 \pm 7.1$ & $<0.001^{* * *}$ & 0.569 \\
\hline IQ score & $67.1 \pm 18.1$ & $78.9 \pm 14.8$ & $<0.001^{* * *}$ & $69.6 \pm 18.4$ & $78.5 \pm 18.4$ & $<0.001^{* * *}$ & 0.180 \\
\hline
\end{tabular}

Values are presented as mean \pm standard deviation.

FMA, Fugl-Meyer Assessment; BBS, Berg Balance Scale; K-MBI, Korean version of Modified Barthel Index; K-MMSE, Korean Mini-Mental State Examination; IQ, intelligence quotient.

${ }^{\text {a) }}$ Compared difference of each score (score at discharge - score at admission) between two groups (brain tumor vs. stroke) by Mann-Whitney U test.

${ }^{* * *} \mathrm{p}<0.001$ when comparing each scores of baseline and discharge within same group by Wilcoxon-signed rank test.

Table 4. Rehabilitation outcomes for benign and malignant brain tumor subgroups

\begin{tabular}{|c|c|c|c|c|c|c|c|}
\hline & \multicolumn{3}{|c|}{ Benign brain tumor $(n=21)$} & \multicolumn{3}{|c|}{ Malignant brain tumor $(n=14)$} & \multirow{2}{*}{$\begin{array}{c}\text { p-value } \\
\text { (between }^{a} \\
\text { groups) }\end{array}$} \\
\hline & Baseline & Discharge & $\begin{array}{l}\text { p-value } \\
\text { (within } \\
\text { groups) }\end{array}$ & Baseline & Discharge & $\begin{array}{l}\text { p-value } \\
\text { (within } \\
\text { groups) }\end{array}$ & \\
\hline FMA score & $36.7 \pm 22.3$ & $51.1 \pm 15.4$ & $<0.001^{* * *}$ & $31.1 \pm 20.8$ & $45.9 \pm 20.7$ & $0.002^{* *}$ & 0.434 \\
\hline BBS score & $19.1 \pm 17.1$ & $38.0 \pm 15.7$ & $<0.001^{* * *}$ & $13.9 \pm 13.0$ & $32.1 \pm 20.1$ & $0.002^{* *}$ & 0.210 \\
\hline K-MBI score & $37.7 \pm 20.2$ & $61.4 \pm 21.6$ & $<0.001^{* * *}$ & $35.9 \pm 15.6$ & $53.8 \pm 18.4$ & $0.016^{*}$ & 0.702 \\
\hline K-MMSE score & $15.5 \pm 7.3$ & $23.5 \pm 5.1$ & $<0.001^{* * *}$ & $19.3 \pm 9.8$ & $22.6 \pm 7.1$ & $<0.001^{* * *}$ & 0.220 \\
\hline IQ score & $63.9 \pm 16.1$ & $79.1 \pm 12.8$ & $0.001^{* *}$ & $71.5 \pm 20.7$ & $78.5 \pm 17.7$ & $0.002^{* *}$ & 0.156 \\
\hline ECOG scale & $3.0 \pm 0.8$ & $2.9 \pm 0.4$ & $<0.001^{* * *}$ & $1.86 \pm 0.7$ & $2.1 \pm 0.7$ & $0.011^{*}$ & 0.175 \\
\hline
\end{tabular}

Values are presented as mean \pm standard deviation.

FMA, Fugl-Meyer Assessment; BBS, Berg Balance Scale; K-MBI, Korean version of Modified Barthel Index; K-MMSE, Korean Mini-Mental State Examination; IQ, intelligence quotient; ECOG, Eastern Cooperative Oncology Group.

${ }^{a)}$ Compared difference of each score (score at discharge - score at admission) between two groups (brain tumor vs. stroke) by Mann-Whitney U test.

${ }^{*} \mathrm{p}<0.05,{ }^{* *} \mathrm{p}<0.01,{ }^{* * *} \mathrm{p}<0.001$ when comparing each scores of baseline and discharge within same group by Wilcoxonsigned rank test. 
(A)

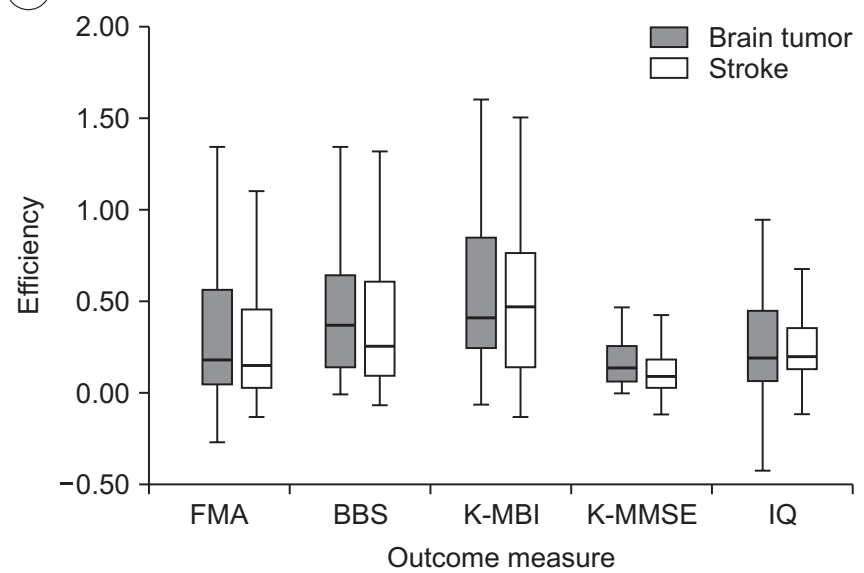

(B)

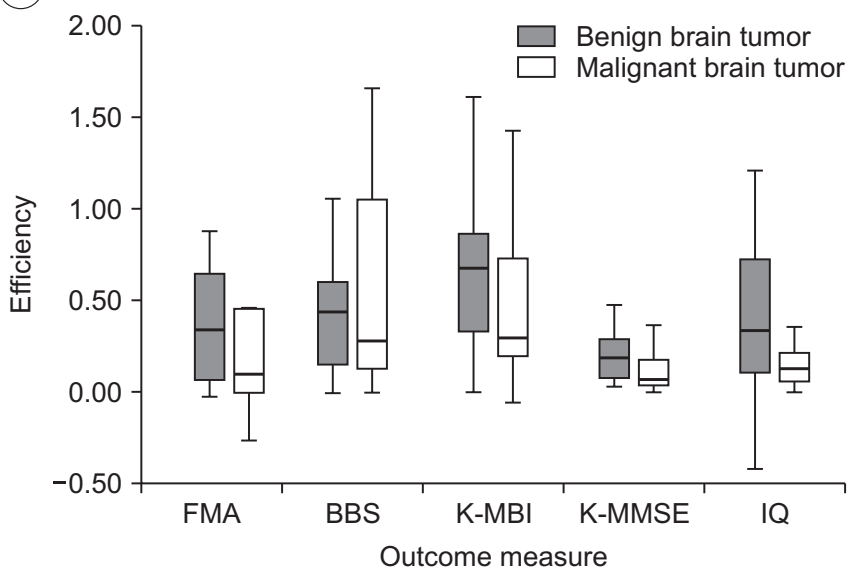

Fig. 2. Comparison of rehabilitation outcome for (A) efficiencies in brain tumor and stroke groups, and (B) efficiencies in malignant and benign brain tumor groups. There is no significant difference when efficiencies between two groups (brain tumor vs. stroke and malignant vs. benign brain tumor) are compared by Mann-Whitney U test. The bold line in the box represents the median value. The interquartile range box represents the middle $50 \%$ of each data. The whisker represents ranges for the bottom 25\% and the top 25\%, excluding outliers. FMA, Fugl-Meyer Assessment; BBS, Berg Balance Scale; K-MBI, Korean version of Modified Barthel Index; K-MMSE, Korean Mini-Mental State Examination; IQ, intelligence quotient.

Table 5. Multivariate linear regression analysis between initial state and rehabilitation outcomes in brain tumor patients

\begin{tabular}{|lllcc}
\hline \multicolumn{1}{c}{ Variable } & \multicolumn{1}{c}{ Outcome } & \multicolumn{1}{c}{ Predictor } & Adjusted R2 & p-value \\
\hline Brain tumor group & Change of FMA & Baseline FMA & 0.032 & $<0.001^{* * *}$ \\
& Change of BBS & Baseline BBS & 0.084 & $0.041^{*}$ \\
& Change of MBI & Baseline K-MBI & 0.354 & $0.034^{*}$ \\
& Change of K-MMSE & Baseline K-MMSE & 0.354 & $<0.001^{* * *}$ \\
\hline & & Baseline IQ & 0.200 & $0.004^{* *}$ \\
\hline Benign tumor subgroup & Change of IQ & Baseline K-MMSE & 0.181 & $0.006^{* *}$ \\
& & Baseline IQ & 0.402 & $<0.001^{* * *}$ \\
\hline & Change of BBS & Baseline K-MMSE & 0.263 & $0.010^{*}$ \\
\hline & Change of K-MBI & Baseline IQ & 0.456 & $<0.001^{* * *}$ \\
\hline & Change of K-MMSE & Baseline BBS K-MBI & 0.150 & $0.047^{*}$ \\
\hline & & Baseline K-MMSE & 0.219 & $0.019^{*}$ \\
\hline Malignant tumor subgroup & Baseline IQ & 0.918 & $0.005^{* *}$ \\
& Change of IQ & Baseline K-MBI & 0.344 & $0.007^{* *}$ \\
& Change of BBS & Baseline FMA & 0.252 & $0.003^{* *}$ \\
\hline & Change of K-MBI & Baseline K-MBI & 0.185 & $0.039^{*}$ \\
\hline & Change of K-MMSE & Baseline K-MBI & 0.373 & $0.029^{*}$ \\
\hline & & Baseline K-MMSE & 0.337 & $0.012^{*}$ \\
\hline
\end{tabular}

FMA, Fugl-Meyer Assessment; BBS, Berg Balance Scale; K-MBI, Korean version of Modified Barthel Index; K-MMSE, Korean Mini-Mental State Examination; IQ, intelligence quotient.

${ }^{*} \mathrm{p}<0.05,{ }^{* *} \mathrm{p}<0.01,{ }^{* * *} \mathrm{p}<0.001$ when comparing each score of baselines and change within the same group by multivariate linear regression analysis. 
was significantly correlated with the corresponding baseline value whereas the improvement in K-MMSE and IQ scores was correlated significantly with both the initial KMMSE score and the initial IQ score.

\section{Long-term survival}

Chart review and telephone survey revealed that 2 of 17 patients $(11.8 \%)$ with benign tumor had expired by the time of the survey, with a mean survival period of 116.6 months since surgery and 112.6 months since initiation of rehabilitation therapy (Fig. 3). Causes of death were not directly associated with brain tumor. They were deemed to be related to old age (83 and 86 years old). Meanwhile, 9 of 13 patients $(69.2 \%)$ with malignant tumor had expired by the time of the survey, with a mean survival period of 52.9 months since surgery and 48.4 months since initiation of rehabilitation therapy (Fig. 3). Their causes of death were tumor recurrence $(n=4)$, tumor progression $(n=3)$, and other medical complications $(\mathrm{n}=2$; pneumonia and myocardial infarction).

\section{Long-term satisfaction}

Twenty-six family caregivers of patients with brain tumors (benign, $n=17$; malignant, $n=9$ ) responded to the telephone survey which was conducted at an average of 2.23 years after patient discharge. Among 26 respondents, family caregivers of 19 survivors (benign tumor, $n=15$;

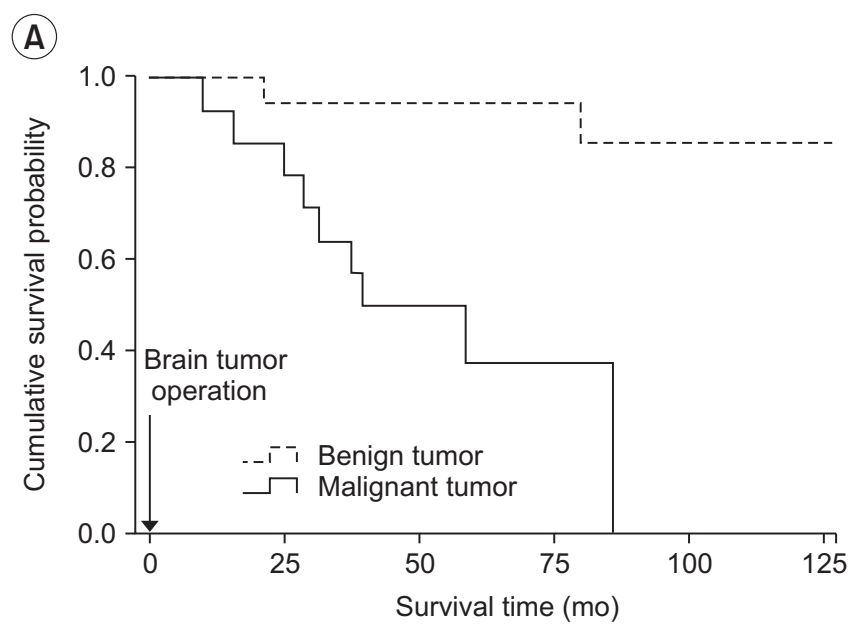

malignant tumor, $\mathrm{n}=4$ ) provided information regarding the long-term functional status (Fig. 4), indicating that functional level had improved or at least been maintained since discharge in most patients (motor function, 89.5\%; cognition, 84.2\%; ADL function, 84.2\%).

Most family caregivers felt that rehabilitation was effective for each functional domain evaluated (motor function, 65.4\%; cognition, 61.6\%; ADL function, 61.6\%) (Fig. 5A). Even when considering only patients with malignant brain tumors $(n=9)$ or those who died $(n=7)$, more than $65 \%$ of family caregivers felt that rehabilitation therapy was helpful for each functional domain (cognition, 77.8\%; ADL function, 77.8\%; motor function, $66.7 \%$ in malignant brain tumor patients and cognition, $71.4 \%$; ADL function, $71.4 \%$; motor function, $85.7 \%$ in deceased patients) (Fig. 5B, 5C).

Approximately $77 \%$ of family caregivers were satisfied with the intensity of rehabilitation therapy, $73.1 \%$ expressed overall satisfaction with the rehabilitation therapy, and $69.2 \%$ stated they would recommend such rehabilitation therapy to patients in a similar situation (Fig. 5A). When considering patients with malignant brain tumors ( $n=9$ ), $88.9 \%$ of family caregivers were satisfied with the intensity of rehabilitation therapy and $77.8 \%$ expressed overall satisfaction, stating that they would recommend such therapy to other patients (Fig. 5B). Even among family caregivers of patients who died $(n=7)$,

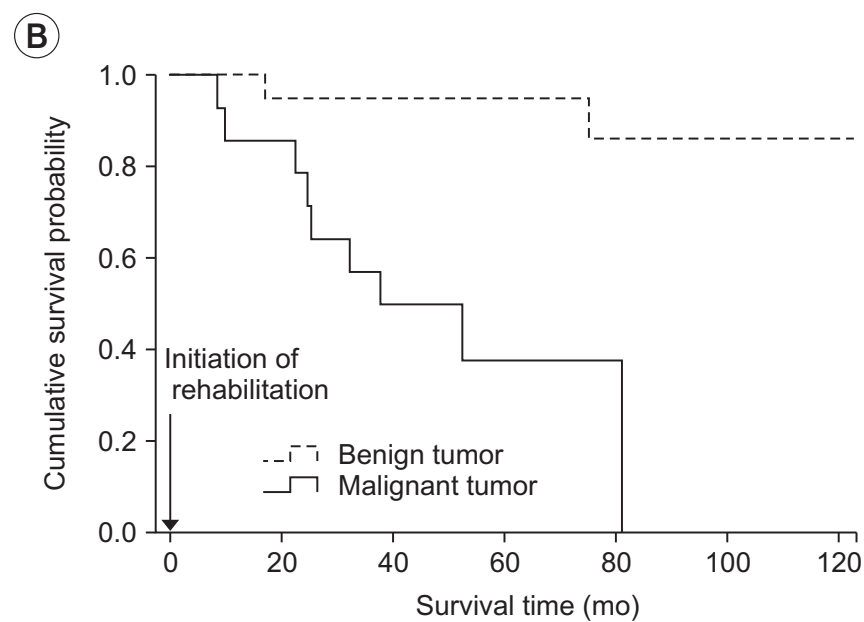

Fig. 3. Survival estimates in patients with brain tumor. The survival rate was analyzed using the Kaplan-Meier survival curve. Cumulative survival probability is drawn according to different pathology (benign vs. malignant) in brain tumor since brain tumor operation (A) and initiation of rehabilitation therapy (B). (A) Since brain tumor operation, overall mean survival was 93.6 months. It was 116.6 months and 52.9 months for benign and malignant tumors, respectively. (B) Since initiation of rehabilitation therapy, overall mean survival was 89.3 months. It was 112.6 months and 48.4 months for benign and malignant tumors, respectively. 
(A)

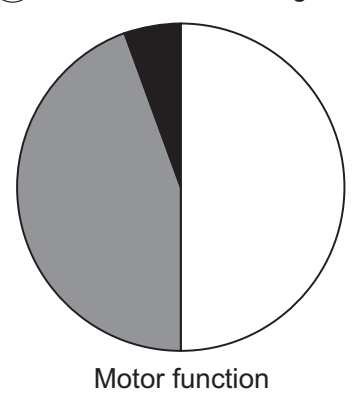

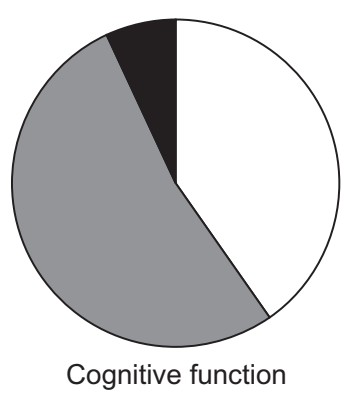

Cognitive function

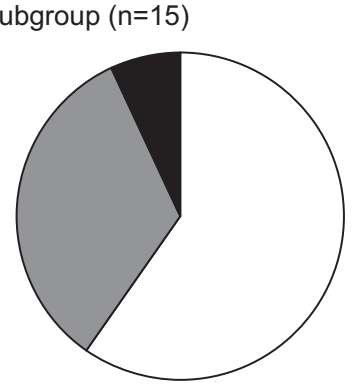

ADL function

Improved $\square$ Similar Worsened

(B)

Current status among the survived patients in malignant brain tumor subgroup $(n=4)$

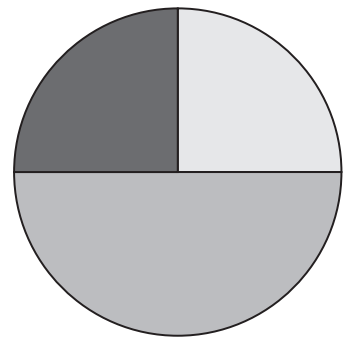

Motor function

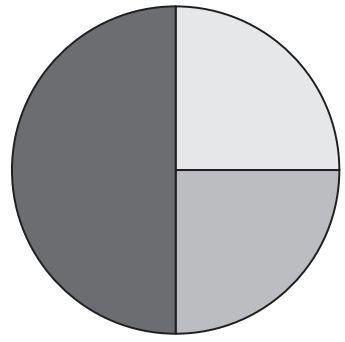

Cognitive function

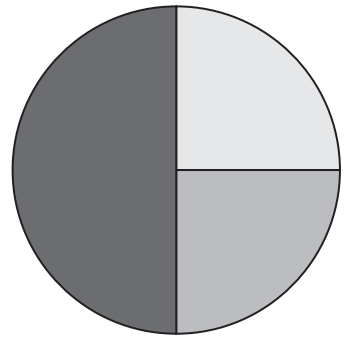

ADL function
Fig. 4. Results of telephone survey about current functions in patients with brain tumor. Opinion of family caregivers on current status of motor, activities of daily living (ADL), and cognitive function compared to the status on discharge in survived patients (3-point scale: improved, similar, and worsened) in benign brain tumor patients $(n=15)(A)$ and malignant brain tumor patients $(n=4)(B)$.
$71.4 \%$ expressed overall satisfaction and $85.7 \%$ were satisfied with the intensity, stating that they would recommend it to other patients (Fig. 5C).

On chi-square test, long-term satisfaction expressed by family caregivers showed significant association with current motor function ( $\mathrm{p}=0.046)$, but not with current cognitive function $(\mathrm{p}=0.069)$, ADL function $(\mathrm{p}=0.314)$, survival status $(\mathrm{p}=0.300)$, or malignancy status $(\mathrm{p}=0.107)$.

\section{DISCUSSION}

In this study, we found that brain tumor patients who received intensive rehabilitation for motor, balance, cognition, and ADL function exhibited significant functional improvement. This improvement was similar to that achieved in patients with stroke. It was not affected by malignancy status. Furthermore, our survey of family caregivers conducted at 1-4 years after intensive rehabilitation suggested that brain tumor survivors had relatively well-maintained status of motor, cognition, and ADL function. Moreover, caregivers of brain tumor patients expressed high rates of long-term satisfaction with the intensive rehabilitation therapy. Despite the relatively high percentage of patients with malignant brain tumors or who died, their satisfaction with the rehabilitation therapy remained high.

The demand for intensive rehabilitation therapy is steadily increasing as treatment options advance. However, intensive rehabilitation therapy appears to be underused in brain tumor patients, especially if the tumor is malignant $[1,2]$. First, although the 5 -year survival rate of patients with malignant brain tumors has improved, it was still as low as $37.5 \%$ in 2011 [4]. Second, recurrence rate is high for malignant brain tumors, with glioblastoma having a recurrence rate of close to $100 \%$ [7]. Due to the low survival rate and high rate of recurrence of malignant brain tumors, physiatrists might be reluctant to prescribe rehabilitation therapy to brain tumor survivors [8].

Sherer et al. [22] have investigated functional recovery in brain tumor patients undergoing rehabilitation and reported functional recovery in 6 of 13 patients. It was later shown that functional recovery was similar between patients with brain tumor and those with stroke in terms 
A Satisfaction survey on rehabilitation therapy in brain tumor group $(n=26)$

1. Did rehabilitation help improve the patient's motor function?

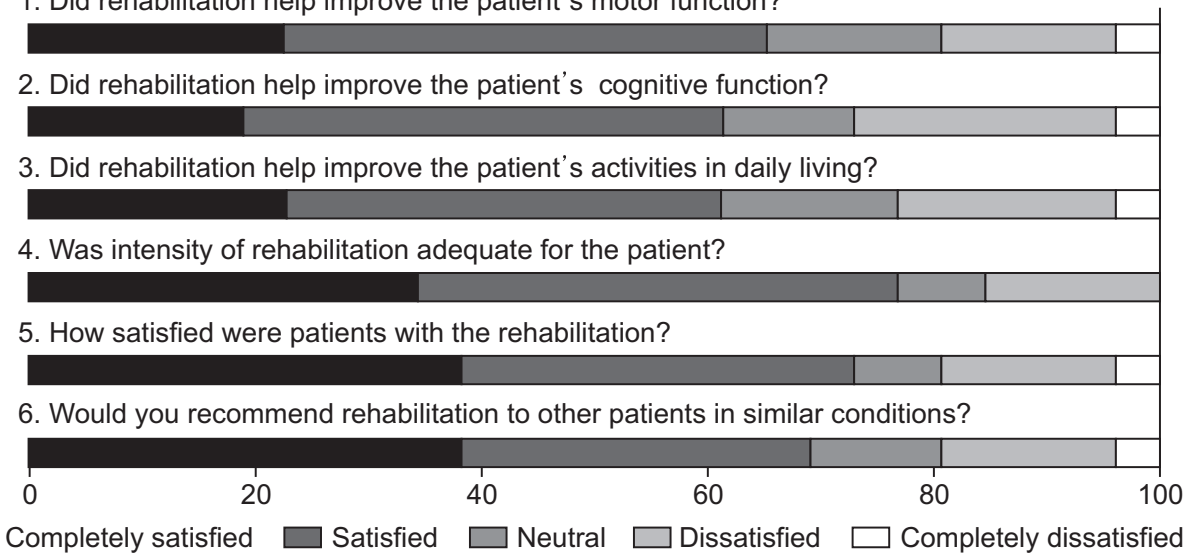

(B) Satisfaction survey on rehabilitation therapy in malignant brain tumor subgroup $(n=9)$

1. Did rehabilitation help improve the patient's motor function?

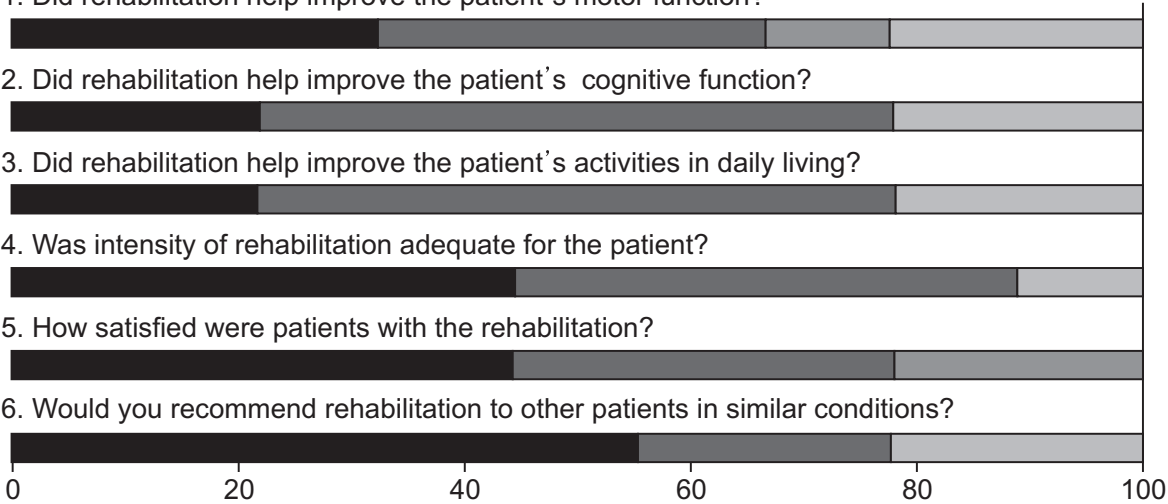

$\square$ Completely satisfied $\square$ Satisfied $\square$ Neutral $\square$ Dissatisfied $\square$ Completely dissatisfied

(C) Satisfaction survey on rehabilitation therapy in deceased patients $(n=7)$

1. Did rehabilitation help improve the patient's motor function?

\begin{tabular}{l|l|l|}
\hline 2. Did rehabilitation help improve the patient's cognitive function? & \multicolumn{1}{|l|}{} \\
\hline 3. Did rehabilitation help improve the patient's activities in daily living? & \\
\hline
\end{tabular}

4. Was intensity of rehabilitation adequate for the patient?

\begin{tabular}{|l|l|}
\hline \\
\hline
\end{tabular}

5. How satisfied were patients with the rehabilitation?

\begin{tabular}{|l|l}
\hline & \\
\hline
\end{tabular}

6. Would you recommend rehabilitation to other patients in similar conditions?

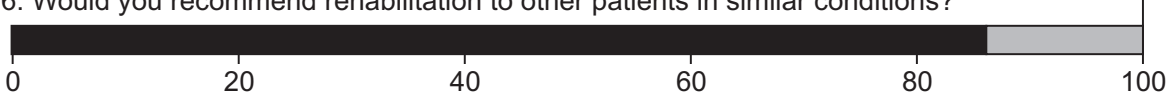

$\square$ Completely satisfied $\square$ Satisfied $\square$ Neutral $\square$ Dissatisfied $\square$ Completely dissatisfied

Fig. 5. Results of telephone survey about satisfaction in patients with brain tumor. (A) Brain tumor patients in total $(\mathrm{n}=26),(\mathrm{B})$ patients with malignant brain tumor $(n=9)$, and (C) deceased patients with both benign and malignant brain tumor $(n=7)$ are depicted. of locomotor function (including balance) [2], cognition [8], and ADL function $[2,11,16]$. These previous results are consistent with observations of the present study, showing that improvements for all evaluated param- eters (FMA score for upper extremity motor function, BBS score for balance, K-MBI score for ADL function, KMMSE and IQ scores for cognition) were similar between brain tumor and stroke patients. In addition, a significant 
improvement in ECOG score was found both in patients with benign tumors and in those with malignant tumors, without showing significant difference between the two subgroups. Since the usefulness of rehabilitation has already been validated in stroke patients, our findings reveal that rehabilitation can help functional improvement following surgery for patients either benign or malignant brain tumor.

Previous studies on the independence of stroke patients employing the Barthel Index and the Functional Independence Measure have reported that poor functional state at admission is associated with poor functional improvement $[23,24]$. Moreover, the initial degree of impairment is known to be a predictor of the effectiveness of rehabilitation for motor function and cognition recovery among stroke patients $[25,26]$. Our present findings confirmed that initial scores of motor, ADL, and cognitive function correlated significantly with their respective outcomes in brain tumor patients.

In this study, we surveyed long-term satisfaction of family caregivers as a surrogate of the long-term evolution of patients' neurological function in order to determine whether rehabilitation could provide meaningful long-term benefits beyond functional improvement achieved in the early period. Several studies have reported the satisfaction with rehabilitation therapy and longterm outcomes among stroke patients [27-29]. However, to the best of our knowledge, no such studies have been conducted on patients with brain tumors. Our present findings suggested that motor function, cognition, and ADL function in patients who received rehabilitation therapy after brain tumor surgery were generally maintained after discharge. Satisfaction levels reported by family caregivers did not differ between the malignant and benign tumor subgroups or between subgroups of survivors and non-survivors. These positive opinions towards rehabilitation therapy and long-term functional outcomes suggest that postoperative rehabilitation is beneficial and necessary for patients with brain tumors regardless of tumor pathology despite their low survival rate.

The satisfaction level expressed by family caregivers with respect to rehabilitation therapy was significantly associated with current motor function, but not with survival status or malignancy status. Among functional domains, motor ability is a significant predictor of func- tional independence in stroke patients [26-30]. In the present study of patients who received intensive rehabilitation therapy following brain tumor surgery, significant short-term improvements were noted in motor function, cognition, and ADL function that were maintained in the long term in $89.5 \%, 84.2 \%$, and $84.2 \%$ of patients, respectively, per results of telephone survey of family caregivers. Among motor, cognition, and ADL function, current motor function seems to have the greatest effect on satisfaction of family caregivers.

This study has several limitations. First, the size of the brain tumor group was smaller than the minimum sample size estimated based on power analysis. Therefore, our investigation is considered a pilot study and findings warrant validation in larger patient populations. Furthermore, we could not perform further analysis of outcomes and satisfaction stratified according to histological type, lesion site, tumor size, or treatment strategy (tumor resection, radiotherapy, and chemotherapy). Second, long-term outcome was determined only by subjective judgment of family caregivers. Although a quantitative assessment of the current functional status would be more appropriate from a purely methodological perspective, the questionnaire-based survey of family caregivers was more appropriate from a practical perspective as some patients died or could not have traveled to the hospital. Third, as the telephone survey was conducted at 1-4 years post-discharge, these data might have been affected by recall bias. Moreover, certain important aspects (such as whether or not patients received additional rehabilitation therapy) were not covered by the survey. Fourth, this study did not include a true control group (i.e., patients who did not receive rehabilitation therapy after brain tumor surgery). Thus, we could not confirm whether the improvement in neurological function was due to natural recovery or rehabilitation.

To conclude, brain tumor patients who received intensive rehabilitation following surgery achieved improvements in the outcomes of motor, cognition, and ADL function similar to those noted in stroke patients, regardless of malignancy status. Moreover, despite the low survival rate and high recurrence rate in patients with malignant brain tumors, their family caregivers expressed a high degree of satisfaction with functional improvements following intensive rehabilitation therapy. These findings suggest that postoperative rehabilitation should be con- 
sidered for patients with brain tumors regardless of tumor pathology as such therapy may be beneficial, despite such patients have a low survival rate.

\section{CONFLICT OF INTEREST}

No potential conflict of interest relevant to this article was reported.

\section{ACKNOWLEDGMENTS}

This research was supported by a grant (No. HI16C1559) of the Korea Health Technology R\&D Project through the Korea Health Industry Development Institute (KHIDI) funded by the Ministry of Health \& Welfare, Republic of Korea.

\section{AUTHOR CONTRIBUTION}

Conceptualization: Kim M, Cho KG. Methodology: Yu J, Jung Y, Park J. Formal analysis: Kim JM, Suh M. Writing original draft: Kim M. Writing - review and editing: Kim M. Approval of final manuscript: all authors.

\section{SUPPLEMENTARY MATERIALS}

Supplementary materials can be found via https://doi. org/10.5535/arm.2019.43.2.129. Table S1. Telephone survey questionnaire for satisfaction on intensive rehabilitation therapy.

\section{REFERENCES}

1. Prados MD, Berger MS, Wilson CB. Primary central nervous system tumors: advances in knowledge and treatment. CA Cancer J Clin 1998;48:331-60.

2. Geler-Kulcu D, Gulsen G, Buyukbaba E, Ozkan D. Functional recovery of patients with brain tumor or acute stroke after rehabilitation: a comparative study. J Clin Neurosci 2009;16:74-8.

3. DeLisa JA. A history of cancer rehabilitation. Cancer 2001;92(4 Suppl):970-4.

4. Jung KW, Yoo H, Kong HJ, Won YJ, Park S, Lee SH. Population-based survival data for brain tumors in Korea. J Neurooncol 2012;109:301-7.

5. Giordana MT, Clara E. Functional rehabilitation and brain tumour patients: a review of outcome. Neurol Sci 2006;27:240-4.

6. Giovagnoli AR, Tamburini M, Boiardi A. Quality of life in brain tumor patients. J Neurooncol 1996;30:71-80.

7. Olar A, Aldape KD. Using the molecular classification of glioblastoma to inform personalized treatment. J Pathol 2014;232:165-77.

8. Han EY, Chun MH, Kim BR, Kim HJ. Functional improvement after 4-week rehabilitation therapy and effects of attention deficit in brain tumor patients: comparison with subacute stroke patients. Ann Rehabil Med 2015;39:560-9.

9. Kirshblum S, O'Dell MW, Ho C, Barr K. Rehabilitation of persons with central nervous system tumors. Cancer 2001;92(4 Suppl):1029-38.

10. Lehmann JF, DeLisa JA, Warren CG, deLateur BJ, Bryant PL, Nicholson CG. Cancer rehabilitation: assessment of need, development, and evaluation of a model of care. Arch Phys Med Rehabil 1978;59:410-9.

11. Huang ME, Cifu DX, Keyser-Marcus L. Functional outcome after brain tumor and acute stroke: a comparative analysis. Arch Phys Med Rehabil 1998;79:1386-90.

12. O'Dell MW, Barr K, Spanier D, Warnick RE. Functional outcome of inpatient rehabilitation in persons with brain tumors. Arch Phys Med Rehabil 1998;79:1530-4.

13. Huang ME, Cifu DX, Keyser-Marcus L. Functional outcomes in patients with brain tumor after inpatient rehabilitation: comparison with traumatic brain injury. Am J Phys Med Rehabil 2000;79:327-35.

14. Marciniak CM, Sliwa JA, Heinemann AW, Semik PE. Functional outcomes of persons with brain tumors after inpatient rehabilitation. Arch Phys Med Rehabil 2001;82:457-63.

15. Huang ME, Wartella JE, Kreutzer JS. Functional outcomes and quality of life in patients with brain tumors: a preliminary report. Arch Phys Med Rehabil 2001;82:1540-6.

16. Greenberg E, Treger I, Ring H. Rehabilitation outcomes in patients with brain tumors and acute stroke: comparative study of inpatient rehabilitation. Am J Phys Med Rehabil 2006;85:568-73.

17. Harvey RF, Jellinek HM, Habeck RV. Cancer rehabilitation: an analysis of 36 program approaches. JAMA 1982;247:2127-31.

18. Haut MW, Haut JS, Bloomfield SS. Family issues in rehabilitation of patients with malignant brain tumors. 
Neurorehabilitation 1991;1:39-47.

19. Filley CM, Kleinschmidt-DeMasters BK. Neurobehavioral presentations of brain neoplasms. West J Med 1995;163:19-25.

20. Kim HJ, Kim DY, Chun MH, Lee SJ. Functional outcomes and characteristics of patients with brain tumors after inpatient rehabilitation: comparison with ischemic stroke. J Korean Acad Rehabil Med 2010;34:290-6.

21. Louis DN, Perry A, Reifenberger G, von Deimling A, Figarella-Branger D, Cavenee WK, et al. The 2016 World Health Organization Classification of Tumors of the Central Nervous System: a summary. Acta Neuropathol 2016;131:803-20.

22. Sherer M, Meyers CA, Bergloff P. Efficacy of postacute brain injury rehabilitation for patients with primary malignant brain tumors. Cancer 1997;80:250-7.

23. Granger CV, Hamilton BB, Gresham GE, Kramer AA. The stroke rehabilitation outcome study. Part II. Relative merits of the total Barthel index score and a fouritem subscore in predicting patient outcomes. Arch Phys Med Rehabil 1989;70:100-3.

24. Alexander MP. Stroke rehabilitation outcome: a potential use of predictive variables to establish levels of care. Stroke 1994;25:128-34.
25. Nijland RH, van Wegen EE, Harmeling-van der Wel BC, Kwakkel G; EPOS Investigators. Presence of finger extension and shoulder abduction within 72 hours after stroke predicts functional recovery: early prediction of functional outcome after stroke: the EPOS cohort study. Stroke 2010;41:745-50.

26. Heruti RJ, Lusky A, Dankner R, Ring H, Dolgopiat M, Barell V, et al. Rehabilitation outcome of elderly patients after a first stroke: effect of cognitive status at admission on the functional outcome. Arch Phys Med Rehabil 2002;83:742-9.

27. Pound P, Gompertz P, Ebrahim S. Patients' satisfaction with stroke services. Clin Rehabil 1994;8:7-17.

28. O'Mahony PG, Rodgers H, Thomson RG, Dobson R, James OF. Satisfaction with information and advice received by stroke patients. Clin Rehabil 1997;11:6872.

29. Musicco M, Emberti L, Nappi G, Caltagirone C; Italian Multicenter Study on Outcomes of Rehabilitation of Neurological Patients. Early and long-term outcome of rehabilitation in stroke patients: the role of patient characteristics, time of initiation, and duration of interventions. Arch Phys Med Rehabil 2003;84:551-8.

30. Han B, Haley WE. Family caregiving for patients with stroke: review and analysis. Stroke 1999;30:1478-85. 


\section{SUPPLEMENTARY MATERIALS}

Table S1. Telephone survey questionnaire for satisfaction on intensive rehabilitation therapy

\begin{tabular}{l}
\multicolumn{1}{c}{ Question } \\
$\begin{array}{l}\text { Completely } \\
\text { agree }\end{array}$ Agree Neutral Disagree $\begin{array}{c}\text { Completely } \\
\text { disagree }\end{array}$ \\
\hline $\begin{array}{l}\text { 1. Did rehabilitation help improve the patient's motor } \\
\text { function? }\end{array}$ \\
2. Did rehabilitation help improve the patient's cognitive \\
function? \\
3. Did rehabilitation help improve the patient's activities in \\
daily living? \\
4. Was intensity of rehabilitation adequate for the patient? \\
5. How satisfied were patients with the overall rehabilitation? \\
6. Would you recommend rehabilitation to other patients in \\
similar conditions?
\end{tabular}

